\title{
Didaktische Funktionen der landeskundlichen Visualisierungen in DaF-Lehrwerken
}

\section{Karolína Pešková}

The study deals with the question what functions of visuals for teaching culture are to be identified in German as a second language textbooks. The focus is put on functions in relation to text, learning process and content. The methodological procedure was based on the analysis of four German as a second language textbooks used in Czech lower secondary schools and six interviews with teachers. The results indicated some deficits and desiderata which are summarized at the end of the study.

German as a second language textbook - visuals for teaching culture - didactic functions textbook analysis - teacher interviews

In dieser Studie wird der Frage nachgegangen, welche Funktionen landeskundliche Visualisierungen in Lehrwerken für den DaF-Unterricht einnehmen. Dabei liegt der Fokus auf Funktionen in Bezug auf den Text, den Lernprozess und den Inhalt. Die methodologische Vorgehensweise der Studie bestand in einer Lehrwerkanalyse von vier in der tschechischen Sekundarstufe I verwendeten DaF-Lehrwerken und sechs Lehrerinterviews. Die Ergebnisse deuteten auf einige Defizite sowie Desiderata hin, die zum Schluss zusammengefasst werden.

DaF-Lehrwerk - landeskundliche Visualisierungen - didaktische Funktionen Lehrwerkanalyse - Lehrerinterviews

Globalisierung, Migration und Integration sind Begriffe, die in letzter Zeit häufig in der öffentlichen Diskussion auftauchen. Die damit verbundenen Themen der kulturellen Pluralisierung und Interkulturalität nehmen nicht nur in den Medien an Bedeutung zu, sondern auch in der sozialwissenschaftlichen bzw. erziehungswissenschaftlichen Fachliteratur (siehe z. B. Zacharaki, Eppenstein und Krummacher 2015; Holzbrecher 2013; Hartung, Nöllenburg und Deveci 2013). Die Fremdsprachendidaktik ist aus dieser Sicht keine Ausnahme, da mit der Ankunft des „cultural turn“ die Entwicklung der interkulturellen Kompetenz als Ergänzung zur kommunikativen Kompetenz im Fremdsprachenunterricht immer stärker akzentuiert wird (siehe z. B. Byram, Holmes und Savvides 2013; Deardorff 2009). So werden auch in der DaF-Didaktik interkulturelle Themen (beispielsweise) im Rahmen des Landeskundeunterrichts immer häufiger diskutiert.

Wegen ihrer Authentizität eignen sich zum Landeskundeunterricht unter anderen verschiedene visuelle oder audiovisuelle Medien, die als kulturelle Produkte verstanden werden, weil sie direkt Einblicke in die jeweilige Kultur eröffnen (vgl. Biechele 2006: 19). In diesem Zusammenhang sind in den letzten Jahren zahlreiche Publikationen erschienen, die Didaktisierungen von literarischen Quellen (z.B. Schweiger 2015), deutschsprachigen Spielfilmen (z. B. Welke und Faistauer 2010; Tonsern 2015) und statischen Visualisierungen (z. B. Badstübner-Kizik 2007, 
2013) bieten. Auch die Arbeit mit sog. neuen Medien wie dem Internet wird nicht ausgeschlossen (z.B. Steinmann 2015; Rösler 2010; Zeuner 2013).

In dieser Studie widme ich meine Aufmerksamkeit den statischen visuellen Medien, konkret den landeskundlichen Visualisierungen (Abbildungen) in DaFLehrwerken, die traditioneller Weise die Basis des Fremdsprachenunterrichts bilden. Dabei gehe ich davon aus, dass im Fremdsprachenunterricht kulturell geprägte Prozesse ablaufen, die das Lernen mit Visualisierungen wesentlich beeinflussen können (vgl. Biechele 2006: 19). Die Visualisierungen vermitteln nicht nur Informationen über die Besonderheiten fremder Zielkulturen, sondern bieten auch Gelegenheiten zur Entwicklung von interkultureller Kompetenz. Der Rezipient weist aufgrund seiner vorherigen Erfahrungen im Augenblick der Wahrnehmung der Visualisierung Sinn und Bedeutung zu und übt zugleich die sogenannte fünfte Fertigkeit des Fremdsprachenlernens aus, nämlich das "Sehverstehen“" (s. Schwerdtfeger 1989).

Die gegenwärtige Situation auf dem Markt für DaF-Lehrwerke in Tschechien führt mich zu der Frage, welche Rolle landeskundliche Visualisierungen in den heutigen DaF-Lehrwerken spielen. Im Vergleich zum deutschsprachigen Raum sind auf dem tschechischen DaF-Lehrwerkmarkt nämlich noch Defizite festzustellen, die auf eine ungenügende Entwicklung der Lehrwerke im interkulturellen Bereich hinweisen. Während in Deutschland ständig neue DaF-Lehrwerke erscheinen, welche die interkulturelle Komponente im Lernprozess berücksichtigen, stehen auf der Liste der Lehrwerke, die vom tschechischen Bildungsministerium für den Unterricht an öffentlichen Schulen zugelassen sind, seit vielen Jahren immer die gleichen tschechischen Lehrwerke. Neuerscheinungen gibt es hier kaum. Dabei zeichnet sich die Tendenz ab, in Tschechien nationale Varianten der neuen deutschen Lehrwerke herauszugeben, die sich z.B. durch tschechische Anleitungen und Erklärungen von den deutschen Originalversionen unterscheiden, wodurch das tschechische Rahmenbildungsprogramm berücksichtigt werden soll ${ }^{1}$. Im Hinblick auf die meisten vorliegenden Lehrwerke in Tschechien möchte ich zugleich auf den Mangel an didaktischer Funktionalität und Reflexion aufmerksam machen, der sich bei vielen landeskundlichen Visualisierungen feststellen lässt. Die Untersuchung der visuellen Komponente von Lehrwerken wird in Tschechien häufig vernachlässigt, und daher sind neue Forschungsinstrumente erforderlich, die ein Sprungbrett für weitere Untersuchungen bilden könnten.

In der vorliegenden Studie möchte ich daher auf die oben genannten Standpunkte und Defizite aufmerksam machen und neue Anregungen zur Forschung im Bereich der Visualisierungen bieten. Als Ausgangspunkt für die weitere Forschung soll diese Studie zur Beschreibung der Visualisierungen beitragen, die in den untersuchten

$1 \mathrm{Zu}$ neuen Lehrwerken dieses Typs gehört beispielsweise das Lehrwerk Beste Freunde (Seuthe, Bovermann, Georgiakaki und Graf-Riemann 2013), das zum DaF-Unterricht (Niveau A1-B1) auf Sekundarstufe I geeignet ist und angibt, den Alltag von Jugendlichen in den Mittelpunkt zu stellen. 
DaF-Lehrwerken vorkommen. Vor allem konzentriere ich mich auf die didaktischen Funktionen der Visualisierungen, wobei ich die wichtigsten Ergebnisse meiner bisherigen Untersuchungen (s. z. B. Pešková und Taušová 2011; Pešková 2014) aus fachdidaktischer Sicht zusammenfasse und ferner entsprechende Empfehlungen für die Praxis formuliere.

\section{Didaktische Grundlegung des fremdsprachlichen Landeskundeunterrichts}

In der Fachliteratur zur Didaktik der Landeskunde werden häufig drei Grundansätze beschrieben: der fotografische/kognitive Ansatz (fokussiert auf die Vermittlung von Wissen über Kultur und Gesellschaft), der kommunikative Ansatz (fokussiert auf die Vermittlung von Kontextwissen und Kompetenzen für die Bewältigung von Alltagssituationen) und der interkulturelle Ansatz (fokussiert auf die Vermittlung von Empathiefähigkeit und Sensibilisierung in interkulturellen Begegnungen). In den letzten Jahren wurden diese drei Ansätze noch um den kulturwissenschaftlichen Ansatz erweitert, der auf einer kritischen Reflexion des Kulturbegriffs aufbaut und an die kulturwissenschaftliche Theorie in den Geistes- und Sozialwissenschaften anknüpft (Schweiger, Hägi und Döll 2015: 4). So basiert der Landeskundeunterricht intensiver auf dem Gedanken, dass Sprachenlernen immer Kulturlernen ist (Krumm 1998: 524), wobei man die beiden Konzepte nicht voneinander trennen kann. Das Ziel eines modernen Landeskundeunterrichts ist dann Fremdverstehen, kulturelle Sensibilisierung, der Abbau von Stereotypen und Klischees sowie die Vermittlung interkultureller (kommunikativer) Kompetenz, die besonders in der englischsprachigen Fachliteratur konzeptualisiert wird (s. z. B. Byram 1997; Deardorff 2004; Spitzberg und Changnon 2009). So werden die Ansprüche an die Fertigkeiten und Kompetenzen der Lernenden immer größer, weswegen die Ziele in der Praxis des Fremdsprachenunterrichts aus verschiedenen Gründen nicht immer erreicht werden können (vgl. Raasch 1997: 71).

Neben den Zielen stellten die geeignete Auswahl der Themen und ihre Präsentation einen wichtigen Ausgangspunkt für den Landeskundeunterricht dar. Eine wichtige Rolle spielen dabei die Lehrwerke, insbesondere im DaF-Unterricht im Ausland (vgl. Földes 1994: 30), wo die Themen oft curricularen Vorgaben unterliegen. Allgemein lassen sich die Themen auf verschiedener Weise bearbeiten deskriptiv, kontrastiv und reflexiv (s. Schweiger, Hägi und Döll 2015:4), was auch verschiedene Formen der Präsentation erfordert.

\section{Visualisierungen in Lehrwerken}

Die vorliegende Studie ist auf statische Visualisierungen eingegrenzt, die sich in Lehrwerken befinden und je nach Typ (z. B. Fotografien, Zeichnungen, Diagramme, 
Piktogramme, Karten) der Veranschaulichung von mannigfaltigen Sachverhalten, Strukturen und Prozessen dienen (weiter s. Pešková 2014: 244). Sie haben eine erhebliche Auswirkung darauf, wie das Lehrwerk insgesamt wahrgenommen wird und wie es seine Funktion erfüllt, besonders was die Interpretation der Lehrwerktexte betrifft (vgl. Eilam und Ben-Peretz 2010: 754). Im Zusammenhang mit dem Text werden verschiedene Funktionen der Visualisierungen unterschieden, die auf bestimmte Weise den Textinhalt präsentieren und damit das Lernen mit dem Text erleichtern. Aus verschiedenen experimentellen Studien (s. Carey und Levin 2002) geht hervor, dass vor allem Visualisierungen mit dekorativer Funktion (ohne direkte Verbindung zum Textinhalt) den geringsten oder gar keinen Effekt haben. Im Unterschied dazu sollen Visualisierungen mit transformierender Funktion zu einem großen Lerneffekt führen: Sie geben die zentrale Lerninformation wieder und rekodieren diese in konkreterer, besser erinnerbarer Form. Die Forschungsbefunde deuten auch darauf hin, dass eine erfolgreiche Verarbeitung der Visualisierung von der dazugehörigen Aufgabe und den Vorkenntnissen der Lernenden abhängt (Elia und Philippou 2004). Da Analysen der Visualisierungen und ihrer Funktionen in Lehrwerken anderer (vor allem naturwissenschaftlicher) Fächer häufiger durchgeführt werden, bleibt eine nähere Beschreibung und Erläuterung dieser Problematik im fremdsprachlichen Kontext weiterhin ein Forschungsdesiderat.

\section{Ziele und methodische Vorgehensweise der Studie}

Obwohl die Forschung im Bereich der Landeskunde relativ intensiv ist, spielen die Analysen von Lehrwerken sowie von Visualisierungen eine eher marginale Rolle (s. z. B. Röttger 2004; Chudak 2007; Maijala 2008; Nguyen 2011). Die vorliegende Studie reagiert auf diese Lücke und setzt sich zum Ziel, die landeskundlichen Visualisierungen in den in Tschechien verwendeten DaF-Lehrwerken aus Sicht ihrer didaktischen Funktionen zu beschreiben. Um die Unterschiede zwischen den tschechischen und deutschen Lehrwerken zu erfassen, habe ich das Ziel mittels folgender Fragestellungen präzisiert:

Welche Funktionen erfüllen landeskundliche Visualisierungen in DaF-Lehrwerken? Wie häufig kommen landeskundliche Visualisierungen in diesen Funktionen in den DaF-Lehrwerken vor?

Unterscheiden sich die aus verschiedenen Verlagen stammenden DaF-Lehrwerke in Bezug auf die Funktionen der landeskundlichen Visualisierungen?

Um die Ergebnisse der Lehrwerkanalyse mit den Anforderungen der Praxis an den Schulen zu vergleichen, wurden die Ansichten von Lehrenden zu den vorhandenen und erwünschten didaktischen Funktionen der landeskundlichen Visualisierungen im Rahmen einer Stichprobe erfragt. Das Ziel der Befragung war es dabei festzustellen, welche didaktischen Funktionen die Lehrenden den landeskundlichen Visualisierungen in DaF-Lehrwerken zuschreiben und wie sie 
diese anhand ihrer Erfahrungen im von ihnen verwendeten DaF-Lehrwerk in Bezug auf ihre erwünschten Funktionen bewerten.

Die Stichprobe bestand aus vier Lehrwerken, die in der tschechischen Sekundarstufe I verwendet werden ${ }^{1}$ und die für den Unterricht von Deutsch als zweiter Fremdsprache geeignet sind. Nach bestimmten Kriterien (Verwendung im Unterricht, verschiedene Verlage, verschiedene Erscheinungsjahre, Orientierung am Erreichen der Niveaustufe A1/A2, zweiter Teil der Lehrwerkreihe ${ }^{2}$ ) wurden zwei Lehrwerke von tschechischen Verlagen, Deutsch mit Max (Fišarová und Zbranková 2007) und Macht mit! (Jankásková et al. 2010), und zwei Lehrwerke von deutschen Verlagen, Planet (Kopp, Büttner und Albert 2005) und Geni@l (Funk et al. 2003) ausgewählt.

Insgesamt wurden 585 Visualisierungen identifiziert, die sich primär auf den Landeskundeunterricht beziehen oder im Rahmen von Aufgaben zur Entwicklung von Fertigkeiten integriert werden, wobei sie zugleich implizit ein landeskundliches Thema betreffen. Die Lehrwerkanalyse erfolgte mittels eines teilweise selbst erstellten Kategoriensystems, das an verschiedene empirische und theoretische Darstellungen anknüpft (z. B. Weidenmann 1991; Byram 1993; Carney und Levin 2002; Biechele 1996; Pýchová 1990)3 . Neben den didaktischen Funktionen erfassen die Kategorien auch die Typen und Inhalte der untersuchten Visualisierungen um eine komplexere Sicht der Visualisierungen zu gewinnen ${ }^{4}$.

Die Funktionen der landeskundlichen Visualisierungen habe ich in drei Gruppen eingeteilt: 1. Funktionen in Bezug auf den Text, 2. Funktionen in Bezug auf den Lernprozess und 3. Funktionen in Bezug auf den Inhalt. Die erste Gruppe umfasst Funktionen, die die Visualisierungen in Bezug auf den Inhalt des Textes haben. So können Visualisierungen den Textinhalt in unterschiedlichem Maße bearbeiten: sie dekorieren, repräsentieren, organisieren, interpretieren oder transformieren ihn. Letztendlich können sie auch keine Funktion in Bezug auf den Text haben, nämlich wenn eine Visualisierung ohne begleitenden Text auftritt (s. Tab.1). Nach dem Typ der Aufgabe (Anleitung zur Aufgabe im Lehrwerk oder Lehrerhandbuch) bzw. der

1 Dabei bin ich von den Ergebnissen einer eigenen kurzen Umfrage in der Sekundarstufe I von verschiedenen Brünner Schulen ausgegangen, die in der Initiationsphase der Untersuchung 2010 durchgeführt wurde. Als das meist benutzte Lehrwerk für Deutsch als zweite Fremdsprache erwies sich das Lehrwerk Deutsch mit Max. Da es in Tschechien üblich ist, an den Grundschulen mehrere Schuljahre das gleiche Lehrwerk in verschiedenen Klassen nacheinander zu benutzen, ist anzunehmen, dass es zwischenzeitlich nur zu unwesentlichen Veränderungen in der Liste der verwendeten Lehrwerke kam.

2 Ich wählte den zweiten Teil der einzelnen Lehrwerkreihen, weil ich angenommen habe, dass für den Zweck der Untersuchung ein etwas höheres Sprachniveau geeigneter ist. Bei höherem Sprachniveau lässt sich vermuten, dass mehr landeskundliche Visualisierungen eingesetzt werden als bei niedrigerem Niveau.

3 Das System wurde durch die Berechnung der Kodierer-Übereinstimmung überprüft (s. Pešková 2014).

4 Das methodische Vorgehen bei der Aufstellung des Kategoriensystems sowie seine ausführliche Beschreibung sind z. B. in Pešková (2014) publiziert. 
Phase des Lernprozesses, in der die Visualisierung erscheint, unterscheide ich eine Aktivierungsfunktion, eine Konstruktionsfunktion, eine Anwendungsfunktion und eine Kontrollfunktion (Tab. 2). Da Visualisierungen auch verschiedene Inhalte präsentieren, die sich in den Funktionen der Visualisierungen widerspiegeln, unterscheide ich in dieser Hinsicht eine Kommunikations-und Sozialisationsfunktion, eine Orientierungs- und Regulationsfunktion, eine propagierende Funktion, eine interkulturelle Funktion, eine stereotypisierende Funktion und eine faktographische Funktion (Tab. 3).

Die erhobenen Daten wurden kodiert und anschließend (mittels Häufigkeitsund Kontingenzanalyse/Chi-Quadrat-Technik) quantitativ bearbeitet um festzustellen, ob das Vorkommen der einzelnen Kategorien in den Lehrwerken unterschiedlich ist (s. Pešková 2014).

\begin{tabular}{|l|l|}
\hline Funktion & Beschreibung der Visualisierung \\
\hline Dekorative Funktion & Sie hat keine direkte Verbindung zum Textinhalt. \\
\hline Repräsentierende Funktion & $\begin{array}{l}\text { Sie veranschaulicht die Akteure, Objekte und Aktivitäten, die } \\
\text { im Text beschrieben oder dargestellt werden. }\end{array}$ \\
\hline Organisierende Funktion & $\begin{array}{l}\text { Sie organisiert den Textinhalt und präsentiert } \\
\text { Zusammenhänge. }\end{array}$ \\
\hline Interpretierende Funktion & $\begin{array}{l}\text { Sie klärt schwer verständliche Textpassagen und erleichtert } \\
\text { das Verständnis komplizierter Begriffe innerhalb dieser } \\
\text { Passagen. }\end{array}$ \\
\hline Transformierende Funktion & $\begin{array}{l}\text { Sie konzentriert sich auf die zentrale Lerninformation und } \\
\text { rekodiert sie in konkreterer und besser erinnerbarer Form. }\end{array}$ \\
\hline Keine Funktion & Sie kommt ohne begleitenden Text vor. \\
\hline
\end{tabular}

Tab. 1: Funktionen in Bezug auf den Text (adaptiert von Carney und Levin 2002)

\begin{tabular}{|l|l|}
\hline Funktion & Beschreibung der Visualisierung \\
\hline Aktivierungsfunktion & $\begin{array}{l}\text { Sie aktiviert das entsprechende Vorwissen, z. B. mittels } \\
\text { Einführung eines neuen Themas. }\end{array}$ \\
\hline Konstruktionsfunktion & $\begin{array}{l}\text { Die Aufgabe für den Lernenden besteht nur im „Lesen“ der } \\
\text { Visualisierung, wobei der Lernende neues Wissen erwerben } \\
\text { kann, das er/sie mit bereits bekanntem Wissen verbindet. }\end{array}$ \\
\hline Anwendungsfunktion & $\begin{array}{l}\text { Sie kommt im Rahmen einer Aufgabe vor, d. h. der Lernende } \\
\text { wird gefordert die Visualisierung z.B. zu beschreiben, } \\
\text { interpretieren, analysieren, zuordnen usw. }\end{array}$ \\
\hline Kontrollfunktion & Sie wird in einem Test oder in einer Kontrollübung umgesetzt. \\
\hline
\end{tabular}

Tab. 2: Funktionen in Bezug auf den Lernprozess (adaptiert von Biechele 1996) 


\begin{tabular}{|l|l|}
\hline Funktion & Beschreibung der Visualisierung \\
\hline $\begin{array}{l}\text { Kommunikations- und } \\
\text { Sozialisationsfunktion }\end{array}$ & $\begin{array}{l}\text { Sie stellt soziale Situationen, Personen in ihrer Umwelt oder } \\
\text { Sachverhalte dar, die der Lernende im Zielland kennenlernen } \\
\text { kann. }\end{array}$ \\
\hline $\begin{array}{l}\text { Orientierungs- und } \\
\text { Regulationsfunktion }\end{array}$ & $\begin{array}{l}\text { Sie ermöglicht mittels Karten oder Pläne die Orientierung } \\
\text { an einem bestimmten Ort oder informiert z. B. mittels } \\
\text { symbolischer Visualisierungen über einen Zustand. }\end{array}$ \\
\hline Propagierende Funktion & $\begin{array}{l}\text { Wird mittels Symbolen, Werbungen, Modellen, Prospekten } \\
\text { usw. realisiert. }\end{array}$ \\
\hline Interkulturelle Funktion & $\begin{array}{l}\text { Sie veranschaulicht eine Form von Interaktion oder die } \\
\text { gegenseitigen Beziehungen zweier oder mehrerer Kulturen. }\end{array}$ \\
\hline Stereotypisierende Funktion & $\begin{array}{l}\text { Sie bietet eine klischeehafte Darstellung von Situationen, } \\
\text { Personen und Sachverhalten. }\end{array}$ \\
\hline Faktographische Funktion & $\begin{array}{l}\text { Sie vermittelt visuelle kulturspezifische und regionalspezifische } \\
\text { Informationen aufgrund realer Daten. }\end{array}$ \\
\hline
\end{tabular}

Tab. 3: Funktionen in Bezug auf den Inhalt (adaptiert von Pýchová 1990)

Um das zweite Ziel der Untersuchung zu erreichen wurde als Methode die qualitative Analyse eines leitfadengestützten Interviews mit Lehrenden an tschechischen Schulen gewählt. Die Stichprobe umfasste sechs tschechische Lehrende (im Folgenden als Lehrende A-F bezeichnet), die in der Sekundarstufe I DaF unterrichten. Alle hatten Erfahrungen mit dem Lehrwerk Deutsch mit Max 2, das sie während der Interviews hinsichtlich bestimmter Aspekte bewerteten. Der Leitfaden des Interviews beinhaltete Kontextfragen zum verwendeten Lehrwerk, Fragen zur Rolle der landeskundlichen Visualisierungen allgemein und zu ihrer Verwendung im Unterricht sowie Fragen zur Bewertung der konkreten landeskundlichen Visualisierungen im jeweiligen Lehrwerk (insbesondere bezüglich Funktionen und wünschenswerten Eigenschaften). Die auf Tonband aufgenommenen Interviews wurden dann transkribiert und nach einem offenen System kodiert.

\section{Ergebnisse der Studie}

\subsection{Funktionen landeskundlicher Visualisierungen anhand der Analyse von Lehrwerken}

Wenn wir uns die Ergebnisse der Lehrwerkanalyse als Ganzes ansehen, lassen sich signifikante Unterschiede zwischen den analysierten Lehrwerken bezüglich der Funktionen der landeskundlichen Visualisierungen, die sie enthalten, feststellen. Dabei ist der Unterschied in allen drei Funktionsgruppen, die ich oben unterschieden habe, statistisch signifikant $\left(\mathrm{p}<0.05^{1}\right)$. Bei genauer Betrachtung lassen sich in den Lehrwerken aber auch einige Gemeinsamkeiten identifizieren.

1 Die einzelnen Werte des Chi-Quadrats waren folgend: im Falle der Funktionen Im Verhältnis zum Text $\mathrm{X}^{2}=55,97$; Im Verhältnis zum Lernprozess $\mathrm{X}^{2}=100,34$; Im Verhältnis zum Inhalt $\mathrm{X}^{2}=70,18$. 
Eine Tendenz, die sich für die ganze Stichprobe als typisch erwiesen hat, ist das häufige Vorkommen von Visualisierungen in darstellender Funktion (insgesamt $61 \%$ der untersuchten Visualisierungen). Visualisierungen in dieser Funktion (am häufigsten Fotografien und Zeichnungen) bezogen sich direkt auf den Textinhalt und stellten die im Text vorkommenden Personen, Gegenstände, Situationen oder Orte dar, was zu einem besseren Textverständnis führen soll. Bedauerlicherweise erschienen in den Lehrwerken auch Visualisierungen, die ohne begleitenden Text vorkamen (insgesamt $20 \%$, davon die meisten in den deutschen Lehrwerken) oder keine direkte Verbindung zum Text besaßen (s. das Beispiel im Anhang). Solche Visualisierungen können zwar einem anderen Zweck dienen, beispielsweise einen Anlass zum freien Sprechen bieten, andererseits können sie aber auch die Aufmerksamkeit der Lernenden ablenken oder völlig funktionslos bleiben. Als interessant, teilweise aber zu erwarten, ließe sich der Befund bezeichnen, dass sich in allen Lehrwerken nur eine geringe Zahl von Visualisierungen in organisierender, interpretierender und transformierender Funktion finden, was wahrscheinlich mit der Dominanz von realistischen Visualisierungen zusammenhängt. Logische (Graphiken, Diagramme) und kartographische Visualisierungen, die größere Ansprüche an die kognitive Verarbeitung stellen, kamen in der ganzen Stichprobe dagegen nur selten vor.

Merkliche Unterschiede zwischen den analysierten Lehrwerken ließen sich bei den Funktionen, die im Zusammenhang mit dem Lernprozess stehen, feststellen. Die häufigste Funktion dieser Gruppe war zwar mit insgesamt 37 \% die Konstruktionsfunktion, aber der Anteil der Visualisierungen in dieser Funktion unterschied sich in den einzelnen Lehrwerken. In den tschechischen Lehrwerken kamen solche Visualisierungen nämlich häufig ohne Aufgabenstellung vor (s. das Beispiel im Anhang), ${ }^{1}$ während die Visualisierungen in den deutschen Lehrwerken gewöhnlich in Aufgaben integriert waren, welche die SchülerInnen zu konkreten Arbeitsaufträgen (z. B. Vergleich der Visualisierung mit dem Inhalt des Textes, Zuordnung der Visualisierung zu bestimmten Absätzen) anleiteten. So überwog in den deutschen Lehrwerken die Anwendungsfunktion (über $30 \%$ ), die eine aktivere Rolle der Lernenden erfordert und vermutlich die Lernwirkung erhöht. Weniger Unterschiede fanden sich bei der Aktivierungsfunktion (insgesamt $25 \%$ der untersuchten Visualisierungen), die v. a. Visualisierungen auf den Einstiegsseiten der Lektionen erfüllen und die LernerInnen auf ein neues Thema einstimmen.

Die in den Lehrwerken am häufigsten vorkommende inhaltliche Funktion war eindeutig die Kommunikations- und Sozialisationsfunktion (insgesamt $54 \%$ der Visualisierungen), was sich durch die hohe Anzahl an kommunikativen Aktivitäten

1 Visualisierungen in dieser Funktion können zwar den Lernenden ermöglichen, ihre Konstruktionen ausgehend von individuellen Wissensbeständen durchzuführen, aber nur unter der Voraussetzung, dass sie den Visualisierungen Aufmerksamkeit widmen oder vom Lehrenden durch Anweisungen bzw. Hilfestellung unterstützt werden. 
sowie alltagsrelevanten und sozialorientierten Themen erklären lässt (z. B. soziale Interaktion und Alltagsleben inkl. Freizeitverhalten von Jugendlichen). Die Anzahl der Visualisierungen in faktographischer Funktion (insgesamt 31 \%) hängt dann mit dem Vorkommen geographischer Themen (z. B. mittels Fotografien präsentierte Sehenswürdigkeiten und touristische Ziele in den deutschsprachigen Ländern; s. das Beispiel im Anhang) eng zusammen. Andere Funktionen wie z. B. die interkulturelle aber auch die sterotypisierende Funktion kamen nur selten vor.

Die Ergebnisse deuten an, dass es eine Verbindung zwischen den Typen und Funktionen der Visualisierungen gibt, was auch statistisch überprüft wurde. ${ }^{1}$ Einige Typen haben also die Tendenz, gleichzeitig mit bestimmten Funktionen vorzukommen: z. B. realistische Visualisierungen (Fotografien) mit darstellender Funktion (auf den Text bezogen), Konstruktionsfunktion (in Bezug zum Lernprozess) und Kommunikations- und Sozialisationsfunktion (auf den Inhalt bezogen). Daraus geht hervor, dass wegen der begrenzten Zahl der Typen auch der Wirkungsbereich der Funktionen begrenzt wird. Obwohl sich Fotografien sehr gut für den Fremdsprachenunterricht eignen (z. B. als Motivationsmittel und zur Widerspiegelung der Realität), wären es empfehlenswert, auch andere Typen im Landeskundeunterricht einzusetzen, die dem Lernenden kognitiv anspruchsvollere Tätigkeiten abverlangen (z. B. die Dekodierung von Symbolen, die Interpretation von Karikaturen, das Suchen von Orten auf einer Karte usw.). Damit würden sich zusätzliche Möglichkeiten zur Reflexion über kulturelle Gegebenheiten eröffnen.

\subsection{Funktionen der landeskundlichen Visualisierungen aus Sicht der Lehrenden}

Die Erkenntnisse, die mittels der Analyse von Lehrwerken gewonnen wurden, wurden durch die Analyse von Interviews mit Lehrenden ergänzt. Die Ergebnisse lassen sich dabei wie folgt zusammenfassen.

Allgemein fassten die befragten Lehrenden landeskundliche Visualisierungen als Motivationsmittel auf, um die SchülerInnen zum Sprechen auf Deutsch und zum Besuch der deutschsprachigen Länder anzuregen: „Die Schüler sehen [mittels Visualisierungen], dass das, was sie lernen, in der realen Welt wirklich zu sehen ist" (Lehrende F). Aus diesem Grund halten die befragten Lehrenden die Motivationsfunktion der Visualisierungen meistens für besonders wichtig. Im Zusammenhang mit der Erhöhung der Motivation hoben sie die Bedeutung der Kommunikations- und Sozialisationsfunktion der Visualisierungen hervor, wobei sie auf die Wichtigkeit der den Alltag der Jugendlichen thematisierenden

1 Der im Rahmen einer Kontingenzanalyse ermittelte Koeffizient von Chuprov sprach für eine mittelstarke Abhängigkeit $(K=0,40$ für Typen und Funktionen im Verhältnis zum Text, $K=0,31$ für Typen und Funktionen im Verhältnis zum Lernprozess, $K=0,39$ für Typen und Funktionen im Verhältnis zum Inhalt; in allen Fällen war p <0,05). 
Visualisierungen hinwiesen. Aus dieser Sicht äußerten sie ihre Zufriedenheit mit dem verwendeten Lehrwerk, wobei sie sich allerdings noch mehr Visualisierungen des Alltags in den deutschsprachigen Ländern wünschen würden, konkret: „... Sie [die Schüler] würden mehr Fotografien der Jugendlichen, Fotografien aus dem Leben ihrer Altersgenossen begrüßen..." (Lehrende A).

Außerdem schrieben die befragten Lehrenden den Visualisierungen eine faktographische bzw. interkulturelle Funktion zu. Sie hielten es für wünschenswert, dass die Lernenden Informationen über das Land der Zielsprache und ihre Einwohner erfahren und sich der Ähnlichkeiten und Unterschiede zwischen verschiedenen Ländern und Tschechien bewusst werden. So war es z. B. für den Lehrenden C wichtig, die Visualisierungen den Lernenden mit folgender Aufgabenstellung auf folgende Weise vorzulegen: "Jetzt siehst du das hier. So sag mir etwas über dich selbst, wie du es machst, ja?"

Weiter sind sich die Lehrenden einig, dass die Visualisierungen, die auf den Einstiegsseiten der Lektionen vorkommen (vgl. Aktivierungsfunktion), der Vorentlastung dienen. Dies wird auch anhand praktischer Erfahrungen mit dem Lehrwerk Deutsch mit Max bestätigt: „Wir sehen uns das Bild an, wir besprechen, worum es in der heutigen Stunde gehen wird, was das Thema der Lektion ist, was die Kinder zum Thema wissen..." (Lehrende C).

Daneben wurde auch die Anwendungsfunktion der Visualisierungen reflektiert. Die Lehrenden betonten den praktischen Umgang mit den landeskundlichen Visualisierungen und erwähnten in diesem Zusammenhang, dass sie wegen des begrenzten Umfangs der im Lehrwerk verfügbaren Aufgaben und Informationen zu den Visualisierungen eigene Aktivitäten entwerfen. Die Lehrende D würde z. B. Folgendes ergänzen: „... sicher mehr Informationen. Zumindest im Lehrerhandbuch, damit man sie [die landeskundlichen Visualisierungen] mehr verwenden kann".

Nach Ansicht der befragten Lehrenden sollte die Visualisierung auch das Textverständnis unterstützen (repräsentieren, was im Text beschrieben ist): „...Das Bild soll bestimmt so aussehen, damit die Kinder den Text einschätzen können“ (Lehrende B). Ungeachtet dieses erwünschten Zustands mangelt es aber laut Aussage der Lehrenden im bewerteten Lehrwerk an kohärenten Texten.

\section{Diskussion und Fazit}

Sowohl die Ergebnisse der Lehrwerkanalyse als auch die Ergebnisse der Lehrerinterviews deuten auf einige Defizite der landeskundlichen Visualisierungen in den ausgewählten DaF-Lehrwerken hin. In allen analysierten Lehrwerken dominiert bei den Visualisierungen die darstellende Funktion, die einen direkten Zusammenhang zwischen Text und Visualisierungen herstellt. Zugleich wurde aber auch ein hoher Anteil von Visualisierungen ohne Funktion in Bezug auf den 
Text identifiziert, was generell betrachtet einen begrenzten Sprach-Input für die Lernenden zur Folge haben kann. In diesem Sinne sprachen sich auch die befragten Lehrenden für eine stärkere Verbindung von Visualisierungen und Text aus.

In Bezug aufden Inhalt erwies sich die Kommunikations-und Sozialisationsfunktion als typisch, die sich durch häufig vorkommende alltagsrelevante und sozialorientierte Themen erklären lässt. Die Aufnahme solcher Visualisierungen in die Lehrbücher wird von den Lehrenden positiv bewertet, sie würden aber sogar noch mehr solche Visualisierungen begrüßen, da sie sich der Bedeutung der Motivation von jugendlichen Lernenden bewusst sind (zur Motivation von Jugendlichen im Deutschunterricht s. Salomo 2014).

Die größten Unterschiede zwischen den deutschen und tschechischen Lehrwerken bestanden bei den auf den Lernprozess bezogenen Funktionen, was an den unterschiedlichen Aufgaben zu den Visualisierungen zu erkennen ist. So können viele Visualisierungen in Lehrwerken funktionslos bleiben, weil im Lehrwerk oder Lehrerhandbuch zu wenig Informationen zum Übungszweck der Visualisierungen enthalten sind. Zugleich sind die Anwendungsmöglichkeiten (sowieso Wahrnehmungsmöglichkeiten) der Visualisierungen in hohem Maße vom Typ der Visualisierung abhängig, was mit dem festgestellten Verhältnis zusammenhängen kann.

In meiner Analyse ging ich von der Annahme aus, dass das Lehrwerk über ein curriculares Potenzial verfügt, dass bewusst oder auch unbewusst von den Unterrichtenden umgesetzt werden kann und verschiedene Interpretationsmöglichkeiten bietet (vgl. Ben-Peretz 1990: 45). In der Unterrichtspraxis bedeutet dies, dass es an dem Lehrenden liegt, ob er/sie sich am Lehrwerk (Aufgaben zu Visualisierungen) und Lehrerhandbuch (Anweisungen zu Aufgaben) orientiert oder ein eigenes Vorgehen wählt. Natürlich können Lehrwerke nicht die ganze Realität des Kulturgegenstandes erfassen, da sie z. B. nicht die Möglichkeit haben, tagesaktuelle Themen einzubeziehen. Deswegen liegt es an dem Lehrenden, weitere Medien und Methoden im Unterricht einzusetzen.

Für die Lehrwerkgestaltung kann man überdies bestimmte Desiderata formulieren. Neben der Verstärkung des Zusammenhangs zwischen Visualisierung und Text sowie einer größeren methodischen Unterstützung bei der Arbeit mit Visualisierungen wäre es empfehlenswert, verschiedene Typen von landeskundlichen Visualisierungen im Rahmen von vielfältigen Aufgaben einzubeziehen. Eine solche Differenzierung kann eine weitreichendere Verwendung von Lehrwerken im Landeskundeunterricht ermöglichen, was Lernenden und Lehrenden helfen kann, neue Rollen und Möglichkeiten des Landeskundeunterrichts zu entdecken z. B. partizipativ (lernendenorientiert), sprach- und kulturbezogen (integrativ), kulturreflexiv, an Auseinandersetzung mit Klischees orientiert, projektorientiert usw. (s. Schweiger, Hägi und Döll 2015: 7-10). 
Die Visualisierungen verstehen sich nämlich nicht von selbst, sondern bedürfen der Interpretation (s. Peiser und Jones 2013). Die Lehrewerkanalyse, die wegen ihrer begrenzten Möglichkeiten deskriptiv aufgefasst wurde, richtete sich auf das Lehrwerk als Produkt und repräsentierte somit nur einen Ausgangspunkt für weitere Untersuchungen. Im nächsten Schritt könnten auch die Erfragungen der Lernenden bezüglich der landeskundlichen Visualisierungen in Lehrwerken einbezogen werden, wodurch sich ein komplexeres Bild von der Verwendung und Lernwirkung von Visualisierungen gewinnen ließe. Nicht zuletzt wäre ratsam, die Stichprobe an Lehrwerken zu erweitern und neue Lehrwerke (sowie ihre multimedialen Komponenten) zu analysieren um sie dann mit den Ergebnissen dieser Studie zu vergleichen.

\section{Literaturverzeichnis}

Badstübner-Kizik, Camilla (2007): Bild- und Musikkunst im Fremdsprachenunterricht. Zwischenbilanz und Handreichungen für die Praxis. Frankfurt am Main, Peter Lang Verlag. Badstübner-Kizik, Camilla (2013): Die Text-Bild-Kombination Filmplakat. In: Convivium. Germanistisches Jahrbuch Polen. S. 55-84.

Ben-Peretz, Miriam (1990): The Teacher-Curriculum Encounter: Freeing Teachers from the Tyranny of Texts. Albany, NY, State University of New York Press.

Biechele, Barbara (1996): Bilder als Kommunikate und Lernmedien im Fremdsprachenunterricht / DaF. In: Informationen Deutsch als Fremdsprache (Info DaF) 23/6/1996. S. 746-757.

Biechele, Barbara (2006): Anmerkungen zum interkulturellen Bildverstehen. In: Interculture Journal 5/1/2006. S. 17-50.

Byram, Michael (1993): Germany. Its Representation in Textbooks for Teaching German in Great Britain. Frankfurt am Main, Diesterweg.

Byram, Michael (1997): Teaching and assessing intercultural communicative competence. Clevedon, Multilingual Matters Ltd.

Byram, Michael / Holmes, Prue / Savvides, Nicola (2013): Intercultural Communicative Competence in Foreign Language Education: Questions of Theory, Practice and Research. In: The Language Learning Journal 41/3/2013. S. 251-253.

Carney, Russel N. / Levin, Joel R. (2002): Pictorial Illustrations Still Improve Students' Learning from Text. In: Educational Psychology Review 14/1/2002. S. 60-61.

Chudak, Sebastian (2007): Lernerautonomie fördernde Inhalte in ausgewählten Lehrwerken $D a F$ für Erwachsene. Überlegungen zur Gestaltung und zur Evaluation von Lehr- und Lernmaterialien. Possener Beiträge zur Germanistik. Frankfurt am Main, Peter Lang.

Deardorff, Darla K. (2004). Intercultural Competence Model. Identification and Assessment of Intercultural Competence as a Student Outcome of Internationalization. Raleigh, North Carolina State University.

Deardorff, Darla K. (2009): The SAGE Handbook of Intercultural Competence. Los Angeles, Sage.

Eilam, Billie / Ben-Peretz, Miriam (2010): Revisiting Curriculum Inquiry: The Role of Visual Representations. In: Curriculum Studies 42/6/2010. S. 751-774.

Elia, Iliada / Philippou, George (2004): The Functions of Pictures in Problem Solving. In: Proceedings of the 28th Conference of the International Group for the Psychology of 
Mathematics Education - 2. Bergen, Bergen University College. S. 327-334.

Fišarová, Olga / Zbranková, Milena (2007): Němčina A1. Deutsch mit Max-2. díl. Učebnice pro základni školy a víceletá gymnázia. Plzeň, Fraus.

Földes, Csaba (1994): Zum Deutschlandbild der DaF-Lehrwerke: von der Schönfärberei zum Frustexport? Ein Diskussionsbeitrag. In: Deutsch als Fremdsprache 32/1/1995. S. 30-32.

Funk, Hermann / Koenig, Michael / Koithan, Ute / Scherling, Theo (2003): Geni@l Němčina jako druhý cizi jazyk. Kursbuch A2. Berlin, München, Langenscheidt.

Hartung, Regine / Nöllenburg, Katty / Deveci, Özlem (Hg.) (2013): Interkulturelles Lernen. Ein Praxisbuch. Schwalbach am Taunus, Wochenschau Verlag.

Holzbrecher, Alfred (Hg.) (2013): Interkulturelle Schule. Eine Entwicklungsaufgabe. Schwalbach am Taunus, Wochenschau Verlag.

Jankásková, Miluše / Dusilová, Doris. / Schneider, Mark / Krüger, Jens (2010): Macht mit! Učebnice némčiny pro základni školy a víceletá gymnázia, druhý cizi jazyk - 2. díl. Praha, Polyglot.

Kopp, Gabriele / Büttner, Siegfried / Albert, Josef (2005): Planet 2: Deutsch für Jugendliche. Kursbuch. Ismaning, Hueber Verlag.

Krumm, Hans Jürgen (1998): Landeskunde Deutschland, D-A-CH oder Europa? Über den Umgangmit Verschiedenheit im DaF-Unterricht. In: Informationen Deutsch als Fremdsprache 5/1998. S. 523-544.

Maijala, Minna (2008): Zwischen den Welten - Reflexionen zu interkulturellen Aspekten im DaF-Unterricht und in DaF-Lehrwerken. In: Zeitschrift für Interkulturellen Fremdsprachenunterricht 13/1/2008. https://zif.spz.tu-darmstadt.de/jg-13-1/docs/ Maijala1.pdf

Nguyen, Minh Thi Thuy (2011): Learning to Communicate in a Globalized World: To What Extent Do School Textbooks Facilitate the Development of Intercultural Pragmatic Competence? In: RELC Journal 42/1/2011. S. 17-30.

Peiser, Gillian / Jones, Marion (2013): The Significance of Intercultural Understanding in the English Modern Foreign Languages Curriculum: A Pupil Perspective. In: The Language Learning Journal 41/3/2013. S. 340-356.

Pešková, Karolína (2014): Inhaltsanalyse der landeskundlichen Visualisierungen in DaFLehrwerken. In: P. Knecht, E. Matthes, S. Schütze, \& B. Aamotsbakken, Methodologie und Methoden der Schulbuch- und Lehrmittelforschung. Bad Heilbrunn, Verlag Julius Klinkhardt. S. 241-253.

Pešková, Karolína / Taušová, Kristýna (2011): Zur Analyse des Lehrwerks Deutsch mit Max. In: Brünner Hefte zu Deutsch als Fremdsprache, 4/1-2/2011. S. 70-90.

Pýchová, Iva (1990): K funkci vizuálií v rozvoji osobnosti žáka. In: Pedagogika 40/6/1990. S. 669-684.

Raasch, Albert (1997): Der Fremdsprachenunterricht hat sich verändert - auch im Fach Französisch!? In: Neusprachliche Mitteilungen aus Wissenschaft und Praxis 2/1997. S. 68-73.

Rösler, Dietmar (2010): Die Funktion von Medien im Deutsch als Fremd - und Deutsch als Zweitsprache-Unterricht. In: H. J. Krumm (Hg.), Deutsch als Fremd-und Zweitsprache. Berlin, Walter de Gruyter. S. 1198-1213.

Röttger, Evelyn (2004): Interkulturelles Lernen im Fremdsprachenunterricht. Das Beispiel Deutsch als Fremdsprache in Griechenland. Hamburg, Verlag Dr. Kovač.

Salomo, Dorothé (2014): Jugendliche lernen anders Deutsch! In: Fremdsprache Deutsch 51/2014. S. 3-9.

Schweiger, Hannes (2015): Kulturelles Lernen mit Literatur - von Anfang an. In: Fremdsprache Deutsch 52/2015. S. 22-27. 
Schweiger, Hannes / Hägi, Sara / Döll, Marion (2015): Landeskundliche und (kultur-) reflexive Konzepte. Impulse für die Praxis. In: Fremdsprache Deutsch 52/2015. S. 3-10.

Schwerdtfeger, Inge Christine (1989): Sehen und Verstehen. Arbeit mit Filmen im Unterricht als Fremdsprache. Berlin, München, Langenscheidt.

Seuthe, Christiane / Bovermann, Monika / Georgiakaki, Manuela / Graf-Riemann, Elisabeth (2013): Beste Freunde A1/1. Kursbuch - Deutsch für Jugendliche. München, Hueber Verlag.

Spitzberg, Brian H. / Changnon, Gabrielle (2009): Conceptualizing intercultural competence. In: Deardorff, Darla K. (Hg.): The SAGE Handbook of Intercultural Competence. Thousand Oaks, SAGE. S. 2-52.

Steinmann, Cornelia (2015): Landeskunde im Netz. In: Fremdsprache Deutsch 52/2015. S. $44-50$.

Tonsern, Clemens (2015): Kulturelles Lernen mit fiktionalem Film. In: Fremdsprache Deutsch 52/2015. S. 28-33.

Welke, Tina / Faistauer, Renate (Hg.) (2010): Lust auf Film heißt Lust aufLernen. Der Einsatz des Mediums Film im Unterricht Deutsch als Fremdsprache. Wien, Praesens.

Weidenmann, Bernd (1991): Lernen mit Bildmedien. Psychologische und didaktische Grundlagen. Weinheim, Beltz.

Zacharaki, Ioanna / Eppenstein, Thomas / Krummacher, Michael (Hg.) (2015): Interkulturelle Kompetenz. Handbuch für soziale und pädagogische Berufe. Schwalbach am Taunus, Wochenschau Verlag.

Zeuner, Ulrich (2013): Landeskunde und neue Medien. Textfassung für einen Vortrag auf der Alumnitagung des Lehrstuhls DaF/TU Dresden im November 2013. 


\section{Anhang}

\section{EINHEIT 2 Wie komme ich ...?}

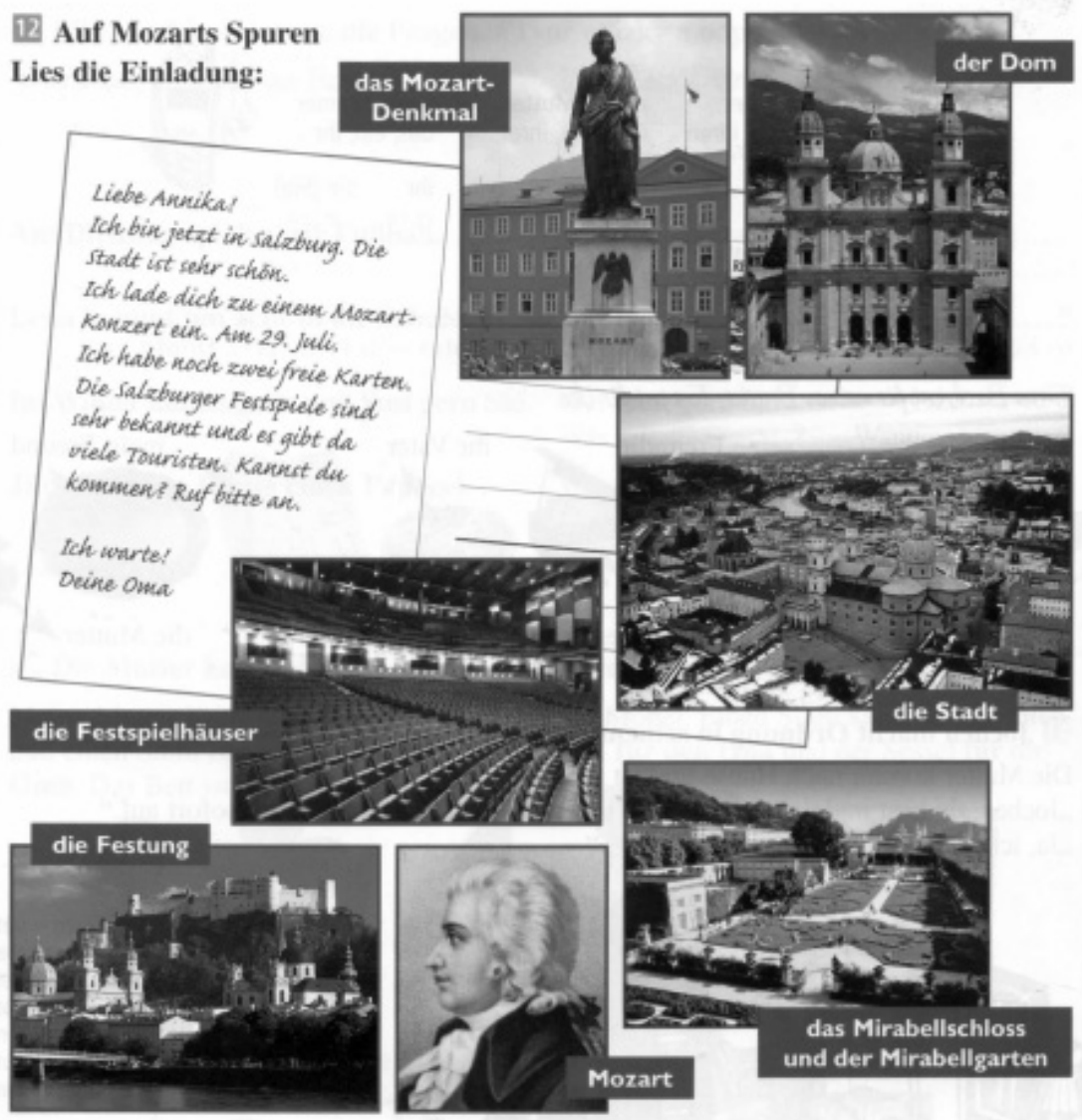

Anmerkung: Die Abbildung kommt aus dem Lehrwerk Deutsch mit Max 2 (Fišarová und Zbranková 2007). Laut des Lehrerhandbuches sollen sich Lernende die Fotos ansehen, Salzburg auf der Karte zeigen und über die Bedeutung des Namens Salzburg sprechen.

Karolína Pešková

Institut výzkumu školního vzdělávání

Pedagogická fakulta Masarykovy univerzity Poříčí 31

60300 Brno

peskova@ped.muni.cz 